\title{
Elektroenzephalografische Befunde bei unterschiedlichen Formen des Status epilepticus - Assoziation mit einzelnen Syndromen und prognostische Bedeutung
}

\author{
Electroencephalographic Patterns in Different Types of Status Epilepticus - Association \\ with Specific Syndromes and Prognostic Significance
}

Autoren

Institut
J. Rösche, K. Rantsch, U. Walter, M. Wittstock, R. Benecke

Klinik und Poliklinik für Neurologie, Universität Rostock
Schlüsselwörter

- Status epilepticus Subtypen

- periodische epileptiforme Entladungen

- rhythmische Delta-Aktivität

Key words

- subtypes of status epilepticus

- periodic epileptiform discharges

- rhythmic delta activity
Bibliografie DOI http://dx.doi.org/ 10.1055/s-0035-1549890 Online-Publikation: 30.7.2015 Klin Neurophysiol 2015; 46: 123-127 (c) Georg Thieme Verlag KG Stuttgart · New York ISSN 1434-0275

Korrespondenzadresse Dr. med., Dr. phil. Johannes Rösche

Klinik und Poliklinik für Neurologie Universitätsmedizin Rostock Gehlsheimerstraße 20 18147 Rostock Johannes.Roesche@med.unirostock.de

\section{Zusammenfassung \\ $\nabla$}

Ziel der Studie: Während der Behandlung eines Status epilepticus abgeleitete elektroenzephalografische Muster sollen hinsichtlich ihrer Assoziation zu bestimmten Subtypen eines Status epilepticus (SE) und ihrer prognostischen Bedeutung untersucht werden.

Methodik: In einer retrospektiven Datenbankanalyse wurden alle Behandlungsepisoden eines SE in der Klinik und Poliklinik für Neurologie der Universität Rostock zwischen dem 01.01.2000 und dem 31.12.2009 identifiziert. Die jeweils ersten EEGs jeder Behandlungsepisode, bei der ein EEG abgeleitet wurde, wurden ausgewertet und die Befunde bezüglich Assoziation zu einzelnen SE Subtypen und dem Outcome, gemessen mit dem Modified Rankin Scale, statistisch untersucht.

Ergebnisse: Bei 35 Behandlungsepisoden eines SE lag wenigstens ein während der Behandlung abgeleitetes EEG vor. Kein EEG-Muster war spezifisch mit einem Status Subtyp assoziiert. Periodische epileptiforme Entladungen waren beim nichtlimbischen Status komplex-fokaler Anfälle und beim Koma infolge eines hypoxischen Hirnschadens häufiger als beim Nonkonvulsiven Status in der postiktalen Phase generalisierter tonisch-klonischer Anfälle. Diese Ergebnisse stimmen nicht mit dem in der Literatur beschriebenen Vorherrschen Periodischer epileptiformer Entladungen beim Nonkonvulsiven Status in der postiktalen Phase generalisierter tonisch-klonischer Anfälle überein. Das Auftreten einer mindestens mittelgradigen Grundrhythmusverlangsamung war mit einer alltagsrelevanten Behinderung assoziiert. Patienten mit Periodischen epileptiformen Entladungen hatten bei Entlassung einen höheren Behinderungsgrad als Patienten ohne solche Muster.

Schlussfolgerung: Das Konzept unterschiedlicher Subtypen des Nonkonvulsiven Status epi-

\section{Abstract}

$\nabla$

Objective: It was planned to investigate whether certain electroencephalographic patterns obtained during the treatment of a status epilepticus (SE) were associated with a particular subtype of SE.

Methods: In a retrospective databank analysis all treatment episodes of a SE between January $1^{\text {st }}$, 2000 and December 31 $1^{\text {st }}, 2009$ in the department of Neurology at Rostock University were identified. The first EEG of each treatment episode, in which an EEG was recorded, was evaluated. Statistical analyses were performed in order to find out whether there is an association of a certain EEG pattern with a particular subtype of SE or whether some EEG patterns signify a worse outcome than others.

Results: In 35 treatment episodes of an SE at least one EEG was obtained during the actual treatment. There was no association of a certain EEG pattern with a particular subtype of SE. Periodic epileptiform discharges were seen more often in non-limbic complex partial status epilepticus and cases of coma due to acute brain injury with epileptiform EEG changes than in non-convulsive status epilepticus (NCSE) in the postictal phase of tonic-clonic seizures. These results are at variance with the published literature, where the frequent occurrence of periodic epileptiform discharges in NCSE in the postictal phase of tonic-clonic seizures is reported. Background slowing with a frequency in the theta range or lower was associated with disability to perform the activities of daily life without any help. On discharge patients with periodic epileptic discharges were more disabled than those without this pattern.

Conclusions: The concept of different subtypes of NCSE needs further evaluation especially with 
lepticus bedarf insbesondere bezüglich therapeutischer Relevanz der weiteren Überprüfung. Spezifische EEG-Befunde bestimmter Statusformen im Erwachsenenbereich sind selten. regard to its therapeutic relevance. In adults EEG patterns specific for a particular subtype of SE are rare.

\section{Einleitung}

Während der Status generalisierter tonisch-klonischer Anfälle (SGTKA) und die Epilepsia partialis continua (EPC) eindeutig klinisch diagnostiziert werden können, erfordert die Diagnose anderer Status Typen grundsätzlich eine Elektroenzephalografie (EEG). Zur elektroenzephalografischen Diagnose eines Nonkonvulsiven Status epilepticus (NKSE) wurden auf einer Consensus-Konferenz in Oxford 2005 [1] 6 Kriterien formuliert ( $\bullet$ Tab. 1). Das sechste hier formulierte Kriterium zeigt, dass in letzter Konsequenz auch beim NKSE die klinische Einschätzung für die Diagnose entscheidend ist. Zur näheren Spezifizierung Periodischer epileptiformer Entladungen nach akuten Hirnschädigungen wurde in der Folge empfohlen, Frequenz und Propagation der Muster in die Beurteilung mit einzubeziehen [2]. Dabei wurde für die elektroenzephalografische Diagnose eines NKSE eine Mindestfrequenz der Periodischen Muster von $1 \mathrm{~Hz}$ gefordert. Zusätzlich sollte entweder eine Propagation in Bezug auf Feld und Frequenz oder fokale motorische Entäußerungen oder eine Frequenz über $2,5 \mathrm{~Hz}$ vorliegen, um die Diagnose eines NKSE zu sichern. Es wurde jedoch auch hier die zusätzliche Möglichkeit gelassen, auch bei Nichtvorliegen dieser Kriterien anhand der Besserung von Klinik und EEG nach Antiepileptikatherapie einen NKSE zu diagnostizieren. Zuletzt wurde der Versuch unternommen, im Rahmen einer umfassenden Übersichts- arbeit spezifische EEG-Muster für einzelne Subtypen des NKSE nach der Klassifikation von Shorvon [3] herauszuarbeiten [4]. Die dabei gefundenen EEG-Muster sind für die Status-Typen, die auch in unserem Kollektiv vorkommen, in 0 Tab. $\mathbf{2}$ dargestellt. Die folgende Studie soll anhand einer retrospektiven Analyse prüfen, ob die unterschiedlichen Subtypen des SE tatsächlich mit unterschiedlichen EEG-Mustern einhergehen und darüber hinaus, ob bestimmten EEG-Mustern eine besondere prognostische Bedeutung zukommt.

\section{Material und Methoden}

$\nabla$

Die hier dargestellten Daten sind Teil einer retrospektiven Aufarbeitung aller Status epilepticus (SE) Behandlungen in der Klinik und Poliklinik für Neurologie der Universität Rostock in der Zeit vom 01.01.2000 bis zum 31.12.2009. Diese Studie wurde von der zuständigen Ethik-Kommission unter der Kennziffer A 201002 genehmigt. Die Patienten wurden durch eine Suche nach den Worten „Status epilepticus“ im elektronischen Archiv der Entlassungsbriefe identifiziert. Dabei wurden 167 Behandlungsepisoden bei 118 Patienten gefunden. Die klinischen Verläufe und Behandlungsergebnisse wurden bereits publiziert [5]. Im Folgenden werden die jeweils ersten EEGs, die während der Behandlung des SE einer Behandlungsepisode abgeleitet

Tab. 1 EEG-Kriterien eines Nonkonvulsiven Status epilepticus (NKSE) nach der Oxford Consensus-Konferenz [1].

1. Häufige oder kontinuierliche fokale elektroencephalografische Anfälle mit iktalen Mustern, die zu- und abnehmen in Bezug auf Amplitude, Frequenz, und/oder räumliche Verteilung.

2. Häufige oder kontinuierliche generalisierte Spike-Wave-Entladungen bei Patienten ohne Anamnese einer vorbestehenden epileptischen Enzephalopathie oder eines anderen Epilepsiesyndroms.

3. Häufige oder kontinuierliche generalisierte Spike-Wave-Entladungen, die eindeutig in Bezug auf Intensität und Frequenz (normalerweise schneller) von einem Vor-EEG bei Patienten mit einer vorbestehenden epileptischen Enzephalopathie oder einem anderen Epilepsiesyndrom abweichen.

4. Periodische lateralisierte epileptiforme Entladungen (PLEDs) oder Bilaterale periodische epileptiforme Entladungen (BIPLEDs), die bei Patienten im Koma in der Folge eines Status generalisierter tonisch-klonischer Anfälle (= subtle status epilepticus) auftreten.

5. Nicht einfach zu interpretierende EEG-Muster: Häufige oder kontinuierliche EEG-Auffälligkeiten (Spikes, Sharp-waves, rhythmische Delta-Aktivität, PLEDs, BIPLEDs, Generalisierte epileptiforme Entladungen (GPEDs), Triphasische Wellen) bei Patienten, deren EEG zuvor keine ähnlichen Auffälligkeiten zeigte nach einem akuten Hirnschaden (hypoxischer Hirnschaden, Meningitis/Enzephalitis, Trauma)

6. Häufige oder kontinuierliche generalisierte EEG-Auffälligkeiten bei Patienten mit epileptischen Enzephalopathien, bei denen ähnliche interiktale Muster bekannt sind, deren klinische Symptome jedoch das Vorliegen eines NKSE nahelegen.

Tab. 2 EEG-Muster bei unterschiedlichen Formen des Nonkonvulsiven Status Epilepticus nach Sutter und Kaplan [4] (auszugsweise).

\section{Statusform}

NKSE bei Lernbehinderung oder anderer Hirnentwicklungsstörung

Limbischer Status komplex fokaler Anfälle

Nichtlimbischer Status komplex fokaler Anfälle

NKSE in der postiktalen Phase generalisierter tonisch-klonischer Anfälle

Koma in der Folge eines hypoxischen Hirnschadens mit epileptiformen EEG-Mustern

\section{EEG-Charakteristik}

Keine spezifische, $\bullet$ Tab. 1

Kontinuierliche oder zu- und abnehmende periodische oder pseudoperiodische Sharp-Wave-Entladungen mit eingelagerten niedrig gespannten Spikes oder Polyspike-Waves mit uni- oder bilateraler frontocentraler Betonung und gewöhnlich langsamer oder supprimierter Hintergrundaktivität oder Ausschließlich zu- und abnehmende rhythmische Delta-Aktivität

Kontinuierliche oder zu- und abnehmende periodische oder pseudoperiodische Sharp-Waves und Spike-Waves mit unilateralem regionalem Beginn und häufiger Anfallsprogression in generalisierte Sharp Wave und slow und/oder supprimierte Wellen gewöhnlich mit frontaler Betonung und langsamer Hintergrundaktivität Generalisierte oder regionale kontinuierliche oder zu- und abnehmende Periodische epileptiforme Entladungen, die PLEDs oder GPEDs mit frontaler Betonung entsprechen und gewöhnlich verlangsamte Hintergrundaktivität Generalisierte zu- und abnehmende Periodische epileptiforme Entladungen die GPEDs entsprechen mit Abschnitten nahezu isoelektrischer Supression der Hintergrundaktivität, die ansonsten gewöhnlich verlangsamt ist. 
Tab. 3 Parameter der EEG-Befundung.

\section{Grundrhythmusverlangsamung}

Regionale Verlangsamung

Fokale epilepsietypische Muster

Generalisierte epilepsietypische Muster

Lateralized Periodic Discharges

Bilateral independent Periodic Discharges

Generalized Periodic Discharges

Stimulus Induced-Evolving Lateralized rhythmic Delta activity
Keine

Keine

Keine

Keine

Keine

Keine

Keine

Keine

$\begin{array}{lll}\begin{array}{l}\text { Leichte } \\ \text { Leichte } \\ \text { Vorhanden }\end{array} & \begin{array}{l}\text { Mittelgradige } \\ \text { Mittelgradige }\end{array} & \begin{array}{l}\text { Schwere } \\ \text { Schwere }\end{array} \\ \begin{array}{lll}\text { Vorhanden } \\ <1 \mathrm{~Hz}\end{array} & 1-2 \mathrm{~Hz} & >2 \mathrm{~Hz} \\ <1 \mathrm{~Hz} & 1-2 \mathrm{~Hz} & >2 \mathrm{~Hz} \\ <1 \mathrm{~Hz} & 1-2 \mathrm{~Hz} & >2 \mathrm{~Hz} \\ \text { Vorhanden } & & \end{array}$

Propagation ja/nein Propagation ja/nein Propagation ja/nein
Tab. 4 Häufigkeit der unterschiedlichen Statusformen in unserem Kollektiv.

\begin{tabular}{lc|}
\hline Statusform & Häufigkeit $\mathbf{n}=$ \\
\hline Status generalisierter tonisch-klonischer Anfälle & 3 \\
\hline Epilepsia partialis continua & 3 \\
NKSE bei Lernbehinderung oder anderer & 1 \\
Hirnentwicklungsstörung & \\
\hline Limbischer Status komplex fokaler Anfälle & 4 \\
\hline Nichtlimbischer Status komplex fokaler Anfälle & 14 \\
\hline $\begin{array}{l}\text { NKSE in der postiktalen Phase generalisierter } \\
\text { tonisch-klonischer Anfälle }\end{array}$ & 7 \\
\hline $\begin{array}{l}\text { Koma in der Folge eines hypoxischen Hirnschadens } \\
\text { mit epileptiformen EEG-Mustern }\end{array}$ & 3 \\
\hline
\end{tabular}

wurden, ausgewertet und die Befunde bezüglich Assoziation zu einzelnen SE Subtypen und dem Outcome gemessen mit dem Modified Rankin Scale (mRS) [6] statistisch untersucht. Dabei wurden Assoziationen mit dem $\mathrm{Chi}^{2}$-Test auf Signifikanz getestet und Gruppenvergleiche mit dem t-Test durchgeführt. Die Auswerteparameter für die EEG-Befunde sind in $\odot$ Tab. 3 dargestellt. Die Auswertung anhand dieser Parameter erfolgte retrospektiv durch den Erstautor. Dabei wurde für die Definition Periodischer epileptiformer Entladungen auf die Leitlinien der American Clinical Neurophysiology Society 2012 zurückgegriffen [7]. Dies bedingt, dass die Terminologie in $\odot$ Tab. 3 geringfügig von der bisher in der Literatur üblichen abweicht. Die Einteilung der Subtypen der NKSE erfolgte nach dem Klassifikationsschema von Shorvon [3].

\section{Ergebnisse \\ $\nabla$}

\section{Kollektivbeschreibung}

Bei 35 Behandlungsepisoden von 24 Patienten (17 weibl./ 7 männl., Alter durchschnittl. 63,1 Jahre, SD 17,3) ) lag mindestens ein während der Statusbehandlung abgeleitetes EEG vor. 4 dieser Patienten verstarben im Verlauf der Behandlung $(=11 \%$ der Behandlungsepisoden, 17\% der Patienten). Der durchschnittliche mRS bei Entlassung war 4,6. Bei 20 Behandlungsepisoden waren die Patienten bei Entlassung bzw. Verlegung in die Rehabilitation schwerstpflegebedürftig ( $m R S=5$ ). Die Häufigkeit der unterschiedlichen Status-Typen ist in $\bullet$ Tab. 4 wiedergegeben.

\section{EEG-Befunde}

Status generalisierter tonisch-klonischer Anfälle. Bei einem von 3 Patienten zeigten sich zum Zeitpunkt der Ableitung fokale epilepsietypische Muster, bei einem weiteren zeigte sich eine mittelgradige Grundrhythmusverlangsamung ohne eindeutige epilepsietypische Muster. Der dritte Patient zeigte faktisch ein Null-Linien-EEG und verstarb im weiteren Verlauf der Behandlung.
Epilepsia partialis continua. Bei allen 3 Patienten fanden sich hochfrequente regionale epilepsietypische Muster, bei zweien auf dem Boden einer mittelgradigen Grundrhythmusverlangsamung. 2 der Patienten hatten zudem eine regionale Verlangsamung in der gleichen Region, wie die epilepsietypischen Muster. NKSE bei Lernbehinderung oder anderer Hirnentwicklungsstörung. Bei dem einzigen Patienten dieser Kategorie fanden sich auf dem Boden einer mittelgradigen Grundrhythmusverlangsamung regionale epilepsietypische Muster mit einer Frequenz über $2 \mathrm{~Hz}$ mit Propagation in Form von Form, Frequenz und Feld ( $\bullet$ Abb. 1).

Limbischer Status komplex fokaler Anfälle. Alle 4 Patienten hatten eine mittelgradige bis schwere Grundrhythmusverlangsamung. 3 dieser Patienten zeigten Generalisierte Periodische Entladungen (GPEDs) mit einer Frequenz um $2 \mathrm{~Hz}$ und einer Propagation. Der verbliebene hatte eine schwere regionale Verlangsamung temporal im Sinne des Vorherrschens von DeltaAktivität mit eingelagerten epilepsietypischen Mustern und eine mindestens mittelgradige Grundrhythmusverlangsamung. Auch einer der Patienten mit GPEDs hatte eine schwere regionale Verlangsamung i.S. von Deltaaktivität.

Nichtlimbischer Status komplex fokaler Anfälle. Knapp 43\% der Patienten hatten in irgendeiner Form Periodische epileptiforme Entladungen im EEG. Weitere knapp 36\% hatten regionale epilepsietypische Muster ( $\bullet$ Abb. 2). Die übrigen Patienten hatten zum Zeitpunkt der Ableitung lediglich eine Grundrhythmusverlangsamung.

NKSE in der postiktalen Phase generalisierter tonisch-klonischer Anfälle. 4 von 7 Patienten hatten regionale epilepsietypische Muster ( $\bullet$ Abb. 3), 2 weitere generalisierte epilepsietypische Muster im EEG. Einer der beiden letzteren besserte sich unter Benzodiazepingabe während des EEGs. Bei dem übrigen Patienten fand sich auf dem Boden einer mittelgradigen Grundrhythmusverlangsamung eine schwere regionale Verlangsamung. Koma in der Folge eines hypoxischen Hirnschadens mit epileptiformen EEG-Mustern. Von 3 Behandlungsepisoden bei 2 Patienten waren 2 mit Generalisierten periodischen Entladungen verbunden, die bei der ersten eine Frequenz von über $2 \mathrm{~Hz}$ hatten und unter Behandlung mit einem Benzodiazepin zurückgingen und bei der zweiten Behandlungsepisode eine Frequenz zwischen $1-2 \mathrm{~Hz}$ hatten und eine Propagation zeigten. Bei der dritten Behandlungsepisode lag zum Zeitpunkt der Ableitung lediglich eine schwere Grundrhythmusverlangsamung vor.

\section{Statistische Auswertung}

Im Gesamtkollektiv ergab sich kein Anhalt für eine statistisch signifikante Assoziation eines bestimmten Schweregrades einer Grundrhythmusverlangsamung oder einer regionalen Verlangsamung oder dem Auftreten von fokalen oder generalisierten epilepsietypischen Mustern mit einem bestimmten Status-Typ. Jedoch wurden bei keiner Behandlungsepisode eines NKSE in 


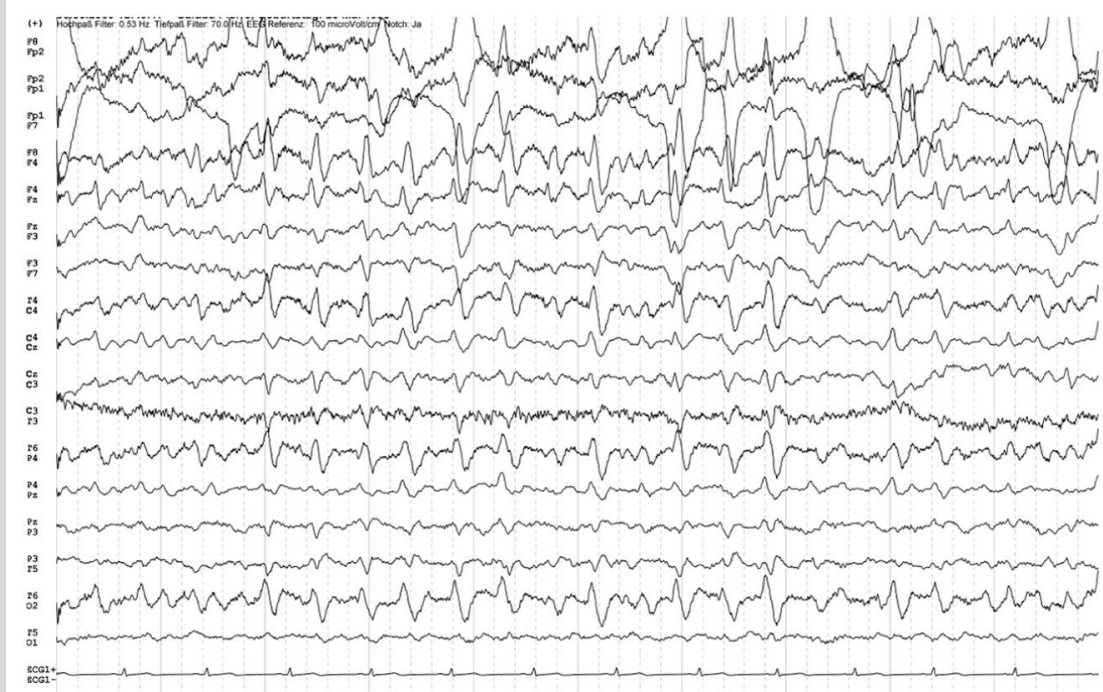

Abb. 1 EEG bei einem Patienten mit NKSE bei Frühkindlichem Hirnschaden.

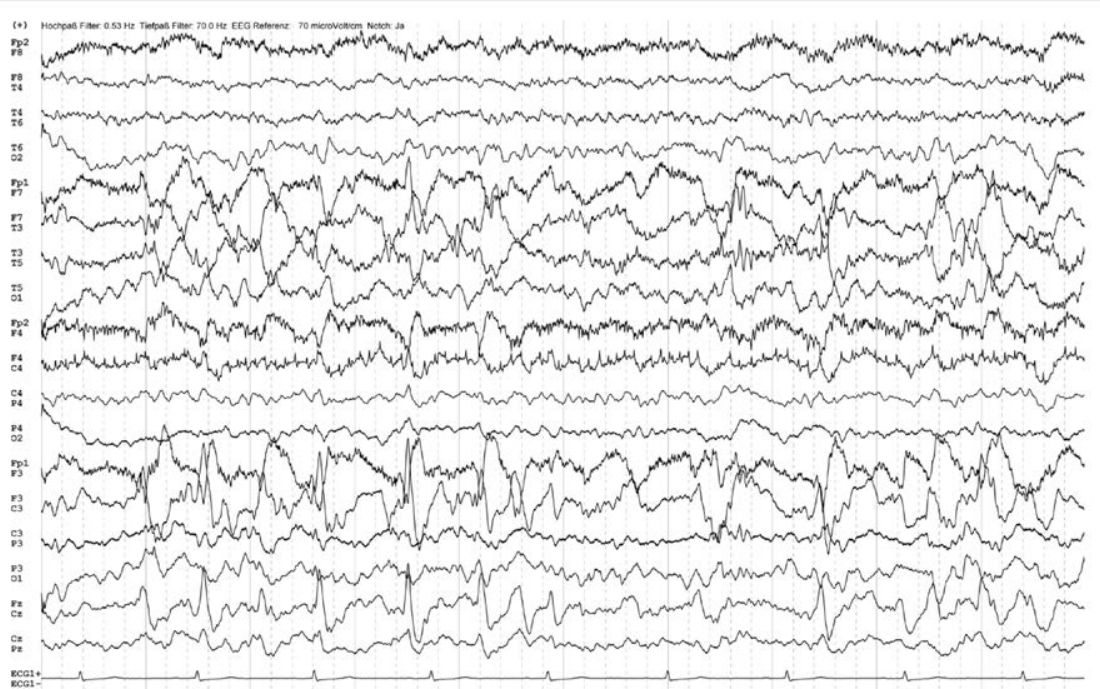

Abb. 2 EEG bei einer Patientin mit nichtlimbischem nonkonvulsivem Status epilepticus und hochfrequenten regionalen epilepsietypischen Mustern.

Abb. 3 EEG bei einer Patientin mit nonkonvulsivem Status epilepticus nach generalisiertem tonisch-klonischen Anfall, der klinisch einer isolierten Aura mit dyskognitiven Symptomen i.S. einer Aphasie entsprach [publiziert als [8]. 
der postiktalen Phase generalisierter tonisch-klonischer Anfälle Periodische Epileptiforme Entladungen gesehen, sodass sich diesbezüglich ein signifikanter Unterschied zu EEGs in Behandlungsepisoden eines nichtlimbischen Status komplex-fokaler Anfälle $(p<0,05)$, EEGs im Koma in der Folge eines hypoxischen Hirnschadens mit epileptiformen EEG-Mustern $(p<0,016)$ und EEGs im limbischen Status epilepticus ergab. Das Auftreten einer mindestens mittelgradigen Grundrhythmusverlangsamung war mit einer alltagsrelevanten Behinderung $(\mathrm{mRS}>\mid=3)$ assoziiert $(p=0,023)$. Patienten mit Periodischen epileptiformen Entladungen hatten bei Entlassung einen höheren Behinderungsgrad als Patienten ohne solche Muster (mRS 5,00 (SD 0,45) vs. 4,4 (SD $1,2) ; \mathrm{p}=0,04)$.

\section{Diskussion \\ $\nabla$}

\section{Kollektiv}

Zunächst überrascht die geringe Zahl der EEG-Ableitungen bei insgesamt 167 Behandlungsepisoden. Nun waren lediglich bei 90 Behandlungsepisoden mehr als 3 Behandlungsschritte (meist 2-malige Benzodiazepingabe und ein i.v. Antiepileptikum) erforderlich [5]. Bei diesen refraktären Verläufen wurde dann immerhin bei gut jeden dritten mindestens ein EEG abgeleitet. Monitor-Überwachungen mit nur 2 EEG-Kanälen, die auf unserer Neurologischen Intensivstation bei den SE-Patienten Standard sind, wurden nicht in die vorliegende Analyse mit einbezogen, da diese Aufzeichnungen nicht mehr verfügbar waren. Die Letalität in der hier analysierten Stichprobe lag etwa anderthalb mal so hoch wie im Gesamtkollektiv [5]. Dies deutet darauf hin, dass schwerpunktmäßig bei schweren refraktären Verläufen ein EEG abgeleitet wurde. Lediglich bei 3 Patienten erfolgte die Ableitung vor Behandlungsbeginn. Bei 2 Patienten mit Status generalisierter tonisch-klonischer Anfälle, 3 Patienten mit nichtlimbischen Status epilepticus, einem mit NKSE in der postiktalen Phase generalisierter tonisch-klonischer Anfälle und einem weiteren mit Koma in der Folge eines hypoxischen Hirnschadens wurden keine elektroenzephalografischen Anfallsäquivalente abgeleitet. Die Ableitungen erfolgten aber, da klinisch der Status aufgrund der fortbestehenden schweren Bewusstseinsstörung klinisch nicht sicher durchbrochen erschien. Daher wurden diese EEGs, die eine Durchbrechung des SE im Vorfeld nahelegen in der Auswertung belassen.

\section{EEG-Befunde}

Bezüglich des NKSE bei Lernbehinderung oder anderer Hirnentwicklungsstörung und des Limbischen Status komplex fokaler Anfälle stimmen unsere EEG-Befunde mit denen von Sutter und Kaplan [4] in der Literatur gefundenen überein. Dagegen entsprachen nicht alle unsere Befunde beim Nichtlimbischen Status komplex fokaler Anfälle und beim Koma nach hypoxischem Hirnschaden den entsprechenden in $\odot$ Tab. 2. Besonders auffällig ist die Diskrepanz beim NKSE in der postiktalen Phase generalisierter tonisch-klonischer Anfälle, wo keines der EEGs in unserem Kollektiv die von Sutter und Kaplan [4] als typisch angesehenen Periodischen epileptiformen Entladungen zeigte. Dies mag zum einen daran liegen, dass wir eindeutig regionale epilepsietypische Muster mit einem Feld von nicht mehr als $2 \mathrm{Ab}$ leitepunkten im 10-20-System trotz periodischem Auftreten nicht als Periodische lateralisierte epileptiforme Entladungen (PLEDs) klassifizierten. Zudem entsprachen die ersten 3 Episoden semiologisch einer isolierten Aura mit kognitiven Symptomen im Sinne einer postiktalen Aphasie (publiziert als [8]). Entsprechend fanden sich auch lediglich regionale epilepsietypische Muster. Bis auf eine Ableitung enthielten jedoch alle elektroenzephalografische Anfallsäquivalente nach den eingangs referierten Kriterien [2]. Damit stellt sich die Frage, ob der NKSE in der Folge generalisierter tonisch-klonischer Anfälle tatsächlich eine eigenständige Kategorie unabhängig von anderen Subtypen des NKSE oder des Subtle-Status in der Spätphase eines Status generalisierter tonisch-klonischer Anfälle darstellt. Letztlich muss sich das Klassifikationsschema von Shorvon [3] in der klinischen Anwendung noch bewähren. Alternativ wurde unlängst ein einfacheres Klassifikationsschema vorgeschlagen, welches die Ätiologie weitgehend ausblendet und lediglich die Unterteilungen „konvulsiv“ und „nonkonvulsiv“ sowie als Subgruppen jeweils ,generalisiert" und „fokal“ enthält. Bei den nonkonvulsiven Status werden sowohl bei „generalisiert" als auch bei „fokal“ weitere 3 Subgruppen differenziert, sodass sich auch hier 6 Subtypen ergeben [9]. Eine Unterscheidung zwischen limbischen und nichtlimbischem Status komplex fokaler Anfälle entfällt aber.

\section{Statistische Auswertung \\ $\nabla$}

Es zeigen sich zumindest in unserem Kollektiv keine spezifisch mit einem Subtyp eines NKSE verbundenen EEG-Muster. Höhergradige Grundrhythmusverlangsamungen und Periodische epileptiforme Entladungen zeigen jedoch offensichtlich eine ausgeprägte diffuse Hirnfunktionsstörung an und sind prognostisch mit einem höheren Behinderungsgrad am Ende der Behandlung assoziiert. Einschränkend muss gesagt werden, dass der AbsenceStatus mit spezifischem EEG-Muster und guter Prognose in unserem Kollektiv nicht vorkam. Insgesamt limitiert die geringe Anzahl an Behandlungsepisoden mit EEG-Ableitung die Aussagekraft dieser Studie. Negative Ergebnisse könnten allein auf der geringen Fallzahl beruhen.

\section{Fazit}

Das Konzept unterschiedlicher Subtypen des Nonkonvulsiven Status epilepticus bedarf insbesondere bezüglich therapeutischer Relevanz der weiteren Überprüfung. Spezifische EEG-Befunde im Erwachsenenbereich sind selten.

Interessenkonflikt: J. Rösche erhielt Vortragsgehälter von UCB $\mathrm{GmbH}$ und EISAI GmbH, war als Berater für EISAI GmbH tätig, erhielt Reisekostenunterstützung von EISAI GmbH und Forschungsgelder von Pfizer $\mathrm{GmbH}$ für ein Investigator initiated Trial. Die übrigen Autoren haben keine Interessenskonflikte. 


\section{Literatur}

1 Walker M, Cross H, Smith S et al. Nonconvulsive status epilepticus: epilepsy research foundation workshop reports. Epileptic Disord 2005; 7: 253-296

2 Herman ST. The electroencephalogram of nonconvulsive status epilepticus. In: Kaplan PW, Drislane FW, Hrsg. Nonconvulsive status epilepticus. New York: Demos Medical Publishing 2009; 41-61

3 Shorvon $S$. What is nonconvulsive status epilepticus, and what are its subtypes? Epilepsia 2007; 48: 35-38

4 Sutter R, Kaplan PW. Electroencephalographic criteria for nonconvulsive status epilepticus: Synopsis and comprehensive survey. Epilepsia 2012; 53: 1-51

5 Rantsch $K$, Walter $U$, Wittstock $M$ et al. Treatment and course of different subtypes of status epilepticus. Epilepsy Research 2013; 107: $156-162$
6 Van Swieten JC, Koudstaal PJ, Visser MC et al. Interobserver agreement for the assessment of handicap in stroke patients. Stroke 1988; 19: 604-607

7 Hirsch LJ, LaRoche SM, Gaspard N et al. American Clinical Neurophysiology Society's standardized critical care EEG terminology: 2012 version. J Clin Neurophysiol 2013; 30: 1-27

8 Rösche J, Schley A, Schwesinger A et al. Recurrent aphasic status epilepticus after prolonged generalized tonic-clonic seizures versus special feature of Todd's paralysis. Epilepsy Behav 2011; 20: 132-137

9 Holtkamp M. Status Epilepticus. Akt Neurol 2012; 39: 429-436 